\title{
Antitumor Effects of a Novel Phenylbutyrate-Based Histone Deacetylase Inhibitor, (S)-HDAC-42, in Prostate Cancer
}

\author{
Samuel K. Kulp, ${ }^{1}$ Chang-Shi Chen, ${ }^{1}$ Da-Sheng Wang, ${ }^{1}$ Ching-Yu Chen, ${ }^{2}$ and Ching-Shih Chen ${ }^{1}$
}

\begin{abstract}
Purpose: To assess the antitumor effects of a novel phenylbutyrate-derived histone deacetylase (HDAC) inhibitor, $(S)$-HDAC-42, vis-à-vis suberoylanilide hydroxamic acid (SAHA) in in vitro and in vivo models of human prostate cancer.

Experimental Design: The in vitro effects of $(S)$-HDAC-42 and SAHA were evaluated in PC-3, DU-145, or LNCaP human prostate cancer cell lines. Cell viability, apoptosis, and indicators of HDAC inhibition were assessed. Effects on Akt and members of the Bcl-2 and inhibitor of apoptosis protein families were determined by immunoblotting. Immunocompromised mice bearing established s.c. PC-3 xenograft tumors were treated orally with $(S)$-HDAC- $42(50 \mathrm{mg} / \mathrm{kg}$ q.o.d. or $25 \mathrm{mg} / \mathrm{kg}$ q.d.) or SAHA ( $50 \mathrm{mg} / \mathrm{kg}$ q.d.) for 28 days. In vivo end points included tumor volumes and intratumoral changes in histone acetylation, phospho-Akt status, and protein levels of $\mathrm{Bcl}-\mathrm{xL}$ and survivin.

Results: (S)-HDAC-42 was more potent than SAHA in suppressing the viability of all cell lines evaluated with submicromolar $I_{50}$ values. Relative to SAHA, $(S)$-HDAC-42 exhibited distinctly superior apoptogenic potency, and caused markedly greater decreases in phospho-Akt, Bcl-xL, and survivin in PC- 3 cells. The growth of PC-3 tumor xenografts was suppressed by $52 \%$ and $67 \%$ after treatment with $(S)$-HDAC- 42 at 25 and $50 \mathrm{mg} / \mathrm{kg}$, respectively, whereas SAHA at $50 \mathrm{mg} / \mathrm{kg}$ suppressed growth by $31 \%$. Intratumoral levels of phospho-Akt and Bcl-xL were markedly reduced in $(S)$-HDAC-42-treated mice, in contrast to mice treated with SAHA.

Conclusions: $(S)$-HDAC-42 is a potent orally bioavailable inhibitor of HDAC, as well as targets regulating multiple aspects of cancer cell survival, which might have clinical value in prostate cancer chemotherapy and warrants further investigation in this regard.
\end{abstract}

The aberrant regulation of core histone acetylation is among the epigenetic modifications that contribute to inappropriate gene expression in cancer cells. The histone deacetylases (HDAC), which participate in this mode of gene regulation, are recognized as promising targets for cancer therapy as they have been linked to the pathogenesis of both solid and hematologic malignancies $(1,2)$. Based on substantial preclinical evidence that HDAC inhibitors can induce growth arrest, differentiation and apoptosis in cancer cells, and suppress tumor growth in mouse models, many structurally distinct, small-molecule HDAC inhibitors are currently undergoing

Authors' Affiliations: 'Division of Medicinal Chemistry and Pharmacognosy, College of Pharmacy, The Ohio State University, Columbus, Ohio and ${ }^{2}$ Department of Family Medicine, College of Medicine, National Taiwan University, and The Gerontology Research Division, The National Health Research Institutes, Taipei, Taiwan

Received 2/27/06; revised 5/23/06; accepted 6/15/06.

Grant support: Public Health Service grants CA94829 and CA112250, and by a grant from theWilliam Randolph Hearst Foundation to C-S. Chen

The costs of publication of this article were defrayed in part by the payment of page charges. This article must therefore be hereby marked advertisement in accordance with 18 U.S.C. Section 1734 solely to indicate this fact.

Requests for reprints: Ching-Shih Chen, College of Pharmacy, 336 Parks Hall, The Ohio State University, 500 West 12th Avenue, Columbus, OH 43210-1291. Phone: 614-688-4008; Fax: 614-688-8556; E-mail: chen.844@osu.edu.

(C) 2006 American Association for Cancer Research.

doi:10.1158/1078-0432.CCR-06-0429 phase I and II clinical evaluations (3-5). The mechanistic paradigm for the anticancer activities of these HDAC inhibitors has been that drug-induced hyperacetylation of core histones leads to chromatin remodeling and reactivated expression of a restricted set of genes regulating cell proliferation, cell cycle progression, and cell survival (3, 4, 6-9). However, emerging evidence suggests that, in addition to this histone acetylationdependent modulation of transcription, the anticancer activities of some HDAC inhibitors may also comprise histone acetylation-independent mechanisms involving nonhistone HDAC substrates $(5,8,10)$, such as nuclear factor $\kappa \mathrm{B}(11,12)$, STAT3 (13), p53 $(14,15)$, and heat shock protein-90 $(16,17)$. More recently, we also showed that broad-spectrum HDAC inhibitors facilitated Akt dephosphorylation by causing the disruption of HDAC-protein phosphatase 1 complexes (18). Together, these findings indicate the complexity in the mechanism of HDAC inhibitors that underlies their high potency in suppressing tumor growth in vitro and in vivo (19).

Among clinically relevant HDAC inhibitors, phenylbutyrate and other short-chain fatty acids are weak inhibitors of HDACs exhibiting millimolar potency in vitro. We recently reported our development of a novel class of potent phenylbutyrate-based HDAC inhibitors possessing submicromolar HDAC-inhibitory activity (20). Further structure-based lead optimization culminated in the generation of $(S)$-HDAC-42, a hydroxamatetethered phenylbutyrate derivative with a low nanomolar $\mathrm{IC}_{50}$ for HDAC inhibition (Fig. 1A; ref. 21). In light of the apparent 
A

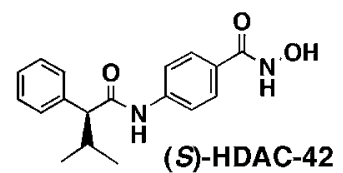<smiles></smiles>

B
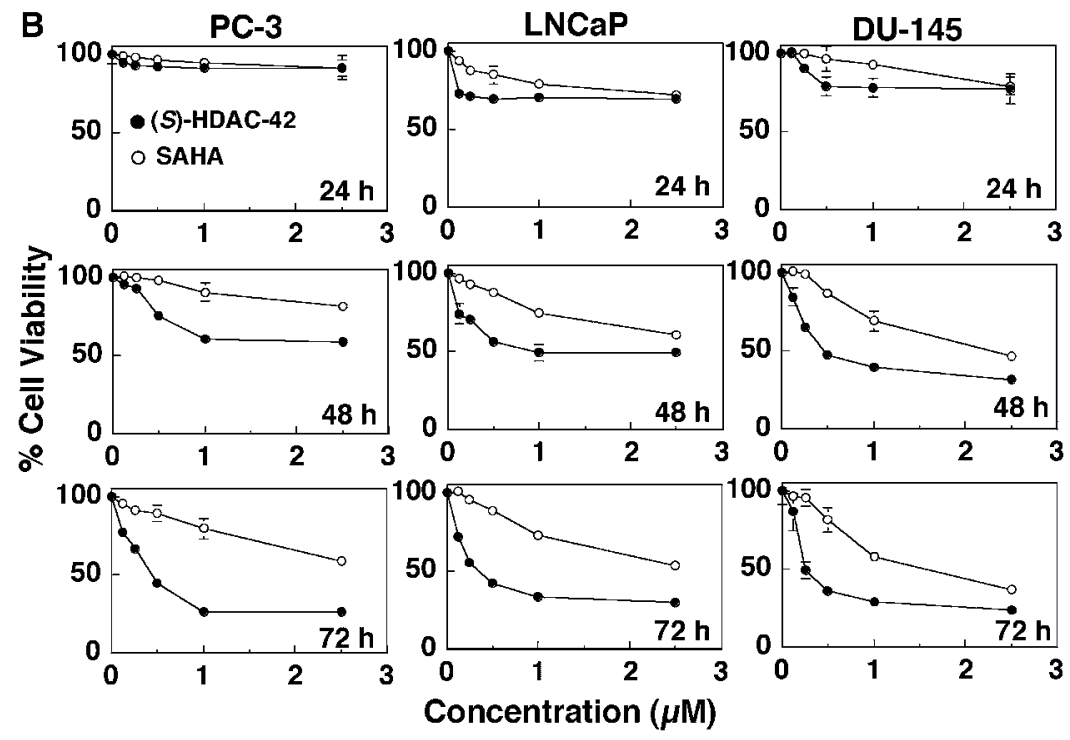

C

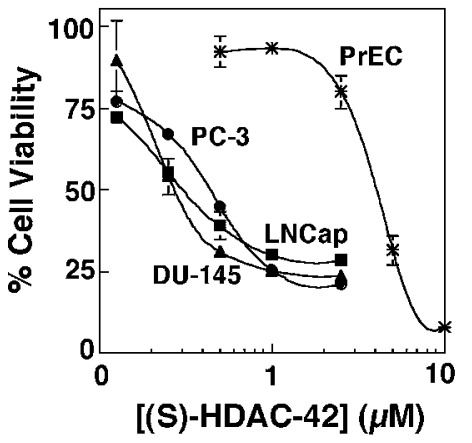

Fig. 1. Antiproliferative effects of $(S)$-HDAC-42 and SAHA in three different prostate cancer cell lines and PrECs. $A$, chemical structures of $(S)$-HDAC-42 and SAHA. $B$, time- and dose-dependent effects of $(S)$-HDAC-42 and SAHA on cell viability in PC-3, LNCaP, and DU-145 cells. Cells were exposed to (S) -HDAC-42 or SAHA at the indicated concentrations in 10\% FBS-supplemented RPMI 1640 in 96-well plates for 24,48 , or 72 hours, and cell viability was assessed by MTTassay. Points, mean; bars, \pm SD $(n=6)$. $C$, effect of $(S)$-HDAC-42 on the viability of PrECs in comparison to PC-3, LNCaP, and DU-145 prostate cancer cell lines. PrECs were treated with $(S)$-HDAC-42 at the indicated concentrations in $10 \%$ FBS-supplemented prostate epithelial growth medium in 96 well-plates for 72 hours, and cell viability was assessed by MTTassay. Corresponding dose-response data for the prostate cancer cell lines were extracted from $(B)$ and presented for purposes of comparison. Points, mean; bars, \pm SD $(n=6)$. role of HDACs in prostate carcinogenesis and progression $(22-25)$, and the efficacy of HDAC inhibitors in preclinical models of prostate cancer $(15,17,26-29)$, the antitumor potential of $(S)$-HDAC-42, in comparison with suberoylanilide hydroxamic acid (SAHA), a HDAC inhibitor currently in clinical trials, was evaluated in prostate cancer models in vitro and in vivo. We show here that $(S)$-HDAC-42 is a potent inhibitor of prostate cancer cell viability and induces a greater apoptotic response relative to SAHA. We also provide evidence that this potent apoptotic activity is associated with the greater ability of $(S)$-HDAC-42 to influence multiple regulators of cell survival including Akt, Bad, Bcl-xL, and members of the inhibitor of apoptosis protein (IAP) family. Finally, these in vitro results were extended to an in vivo prostate cancer xenograft model in which orally administered $(S)$-HDAC-42 potently inhibited tumor growth in association with intratumoral histone hyperacetylation and reductions in phosphoAkt and Bcl-xL levels. (S)-HDAC-42 is a novel orally bioavailable, phenylbutyrate-based HDAC inhibitor with antitumor activity at multiple cellular levels which, based on our results, may have value in prostate cancer chemotherapy and warrants further investigation in this regard.

\section{Materials and Methods}

Reagents. The HDAC inhibitors SAHA and (S)-(+)-N-hydroxy-4(3-methyl-2-phenyl-butyrylamino)benzamide [( $S$ )-HDAC-42] were synthesized in our laboratory with purities exceeding $99 \%$ as shown by nuclear magnetic resonance spectroscopy (300 MHz). ( $S$ )-HDAC-42 (NSC 736012) is a novel hydroxamate-tethered phenylbutyrate derivative $(20,21)$ and is currently undergoing preclinical evaluation through the Rapid Access to Intervention Development Program at the National Cancer Institute. For in vitro studies, stock solutions of inhibitors were prepared in DMSO and diluted in 10\% serumcontaining culture medium for treatment of cells (final concentration of DMSO, $<0.1 \%$ ). For in vivo studies, (S)-HDAC-42 and SAHA were prepared as suspensions in vehicle $(0.5 \%$ methylcellulose $\mathrm{w} / \mathrm{v}$, $0.1 \%$ Tween $80 \mathrm{v} / \mathrm{v}$, in sterile water) for oral administration to xenograft-bearing athymic nude mice. Rabbit antibodies against Akt, $\mathrm{p}-{ }^{473}$ Ser-Akt, p- ${ }^{308}$ Thr-Akt, Bad, p- ${ }^{136}$ Ser-Bad, Bax, Bcl-xL, Bcl-2, caspase 9 , and poly(ADP-ribose)polymerase (PARP) were purchased from Cell 
Signaling Technology, Inc. (Beverly, MA). Mouse antibodies against p21 (clone F-5) and rabbit antibodies against cytochrome $c$ (H-104), ILP (H-202) and cIAP-2 (H-85) were obtained from Santa Cruz Biotechnology, Inc. (Santa Cruz, CA). Rabbit antibodies against cIAP-1 (AF818) and survivin (AF886) were purchased from R\&D Systems, Inc. (Minneapolis, MN), and against acetylated histone $\mathrm{H} 3$ (06-599) from Upstate Biotechnology, Inc. (Lake Placid, NY). Mouse antibodies against $\beta$-actin (clone C4; 69100) were obtained from ICN Biomedicals (Irvine, CA).

Cell culture. The androgen-insensitive PC-3 and DU-145, and androgen-sensitive LNCaP human prostate cancer cell lines were purchased from American Type Culture Collection (Manassas, VA) and cultured in RPMI 1640 (Life Technologies, Grand Island, NY) supplemented with $10 \%$ fetal bovine serum (FBS; Life Technologies). Normal prostate epithelial cells (PrEC) were obtained from Cambrex Bioscience-Walkersville, Inc. (Walkersville, MD) and maintained in the vendor's recommended defined prostate epithelial growth medium. All cell types were cultured at $37^{\circ} \mathrm{C}$ in a humidified incubator containing $5 \% \mathrm{CO}_{2}$. Cells in log phase growth were harvested by trypsinization for use in viability assays and in vivo studies.

Cell viability assay. Cell viability was assessed by using the 3-(4,5dimethylthiazol-2-yl)-2,5-diphenyltetrazolium bromide (MTT; Tokyo Chemical Industry, Inc., Tokyo, Japan) assay in six replicates (96-well format). Cancer cells and PrECs were seeded at 2,500 to 3,000 and 8,000 cells per well, respectively, in 96-well flat-bottomed plates. Twenty-four hours later, cells were treated with HDAC inhibitors at the indicated concentrations in 10\% FBS-supplemented RPMI 1640 or $10 \%$ FBS-prostate epithelial growth medium. The control cells received DMSO at a concentration equal to that in drug-treated cells. At the indicated intervals, $1 / 10$ volume of $10 \times$ MTT $(5 \mathrm{mg} / \mathrm{mL})$ was added to each well and cells were incubated at $37^{\circ} \mathrm{C}$ for 2 hours. Medium was removed and the reduced MTT dye was solubilized in $200 \mu \mathrm{L} /$ well DMSO. Absorbance was determined at $570 \mathrm{~nm}$. Concentrations of compounds that inhibited viability by $50 \%\left(\mathrm{IC}_{50}\right)$ were determined by the median effect method of Chou and Talalay (30) using CalcuSyn software (Biosoft, Ferguson, MO).

Apoptosis assay. Drug-induced apoptotic cell death was assessed using the Cell Death Detection ELISA kit (Roche Diagnostics, Mannheim, Germany), which quantitates cytoplasmic histone-associated DNA fragments in the form of mononucleosomes or oligonucleosomes. Cells were seeded and incubated at 50,000 cells per well in 12-well flat-bottomed plates in 10\% FBS-supplemented RPMI 1640. After 24 hours, cells were treated with HDAC inhibitors at the indicated concentrations and durations. Both floating and adherent cells were collected and the assay was done according to the manufacturer's instructions.

Immunoblotting. Lysates of PC-3 cells treated with HDAC inhibitors at the indicated concentrations for 72 hours were prepared for immunoblotting of acetylated histone $\mathrm{H} 3, \mathrm{p} 21$, actin, cytochrome $c$, caspase 9, PARP, p- ${ }^{473}$ Ser-Akt, p- ${ }^{308}$ Thr-Akt, Akt, p- ${ }^{136}$ Ser-Bad, Bad, Bcl-xL, Bax, survivin, CIAP-1 and CIAP-2, and ILP. Western blot analysis was done as previously reported (18). For immunoblotting of biomarkers in PC-3 tumor xenografts, tumor tissue homogenates were prepared and immunoblotting done as previously described $(18,31)$.

Reverse transcriptase-PCR. Semiquantitative reverse transcriptionPCR was done on total RNA isolated from PC-3 cells treated for 72 hours with the indicated concentrations of $(S)$-HDAC-42 or SAHA using the RNeasy Mini Kit (Qiagen, Inc., Valencia, CA). Following DNase I treatment, $1 \mu \mathrm{g}$ of RNA was reverse-transcribed using Omniscript RT (Qiagen) and oligo(dT). The minimum number of PCR cycles required to obtain a clear signal from the amplicon within the linear amplification phase was determined for each primer set. The following specific primer pairs were used: 5'-GCA TTG TTC CCA TAG AGT TCC-3' and 5'-CAT GGC AGC AGT AAA GCA AG-3' for Bcl-xL; 5'-AAG AAC TGG CCC TTC TTG GA- $3^{\prime}$ and $5^{\prime}$-CAA CCG GAC GAA TGC TTT TT- 3 ' for survivin; and 5'-TCT ACA ATG AGC TGC GTG TG-3', and $5^{\prime}$-GGT CAG GAT CTT CAT GAG GT-3' for $\beta$-actin.
In vivo studies. Intact male $\mathrm{NCr}$ athymic nude mice (5-7 weeks of age) were obtained from the National Cancer Institute (Frederick, MD). The mice were group-housed under conditions of constant photoperiod (12 hours light/12 hours dark) with ad libitum access to sterilized food and water. All experimental procedures using these mice were done in accordance with protocols approved by the Institutional Laboratory Animal Care and Use Committee of The Ohio State University.

Each mouse was inoculated s.c. with $5 \times 10^{5}$ PC-3 cells in a total volume of $0.1 \mathrm{~mL}$ serum-free medium containing 50\% Matrigel (BD Biosciences, Bedford, MA) under isoflurane anesthesia. As tumors became established (mean starting tumor volume, $145.1 \pm 22.1 \mathrm{~mm}^{3}$ ), mice were randomized to four groups $(n=7)$ that received the following treatments: $(a)(S)$-HDAC-42 at $25 \mathrm{mg} / \mathrm{kg}$ body weight q.d., (b) (S)-HDAC-42 at $50 \mathrm{mg} / \mathrm{kg}$ q.o.d., (c) SAHA at $50 \mathrm{mg} / \mathrm{kg}$ q.d., and (d) the methylcellulose/Tween 80 vehicle. Mice received treatments by gavage $(10 \mu \mathrm{L} / \mathrm{g}$ body weight) for the duration of the study. Tumors were measured weekly using calipers and their volumes calculated using a standard formula: width ${ }^{2} \times$ length $\times 0.52$. Body weights were measured weekly. At the conclusion of the study, tumors were harvested, snap-frozen in liquid nitrogen, and stored at $-80^{\circ} \mathrm{C}$ until needed for Western blot analysis of relevant biomarkers. The percentage of reductions in tumor growth were calculated using the formula: $\left[1-\left(T_{\mathrm{f}}-T_{\mathrm{i}}\right) /\left(C_{\mathrm{f}}-C_{\mathrm{i}}\right)\right] \times 100$, where $T_{\mathrm{f}}$ and $T_{\mathrm{i}}$ are the final and initial mean tumor volumes, respectively, of the group receiving a specified treatment, and $C_{\mathrm{f}}$ and $C_{\mathrm{i}}$ are the final and initial mean tumor volumes, respectively, of the control group. One mouse from each treatment group was submitted to The Ohio State University Mouse Phenotyping Shared Resource for evaluation of gross and histologic pathology.

Statistical analysis. Tumor volume data met the assumptions of normality and homogeneity of variance for parametric analysis; thus, group means at 28 days of treatment were compared using one-way ANOVA followed by Fisher's least significant difference method for multiple comparisons. Tumor growth data are expressed as mean tumor volumes \pm SE. In vitro data are expressed as mean \pm SD and were assessed by one-way ANOVA and Fisher's least significant difference method for post hoc comparisons. Differences were considered significant at $P<0.05$. Statistical analysis was done using SPSS for Windows (SPSS, Inc., Chicago, IL).

\section{Results}

(S)-HDAC-42 induces apoptosis in prostate cancer cells. The antitumor effects of $(S)$-HDAC-42 vis-à-vis SAHA were assessed in three different human prostate cancer cell lines, PC-3, DU145 , and LNCaP. These cells were exposed to individual agents over the dose range of 0.1 to $2.5 \mu \mathrm{mol} / \mathrm{L}$, and the cell viability was determined by the MTT assay at 24,48 , and 72 hours. Both agents showed a time-dependent reduction in cell viability. Neither $(S)$-HDAC-42 nor SAHA showed significant antiproliferative effect until 48 hours, and $(S)$-HDAC-42 was clearly more potent than SAHA in suppressing cell viability in all cell lines evaluated (Fig. 1B). All cell lines were comparably susceptible to the antiproliferative effect of $(S)$-HDAC-42 with submicromolar $\mathrm{IC}_{50}$ values after 72 hours of treatment (PC-3, $0.48 \pm 0.02 ; \mathrm{LNCaP}, 0.30 \pm 0.07$; DU-145, $0.43 \pm 0.05$ $\mu \mathrm{mol} / \mathrm{L} ; P>0.8$, ANOVA), irrespective of the differences in androgen responsiveness, the functional status of p53 and PTEN, and the Bcl-xL expression levels that distinguish these cell lines. On the other hand, SAHA exhibited a statistically significant differential effect on growth inhibition among all the cell lines tested, with DU-145 cells being the most sensitive $\left(\mathrm{IC}_{50}, 1.62 \pm 0.16 \mu \mathrm{mol} / \mathrm{L}\right)$, followed by $\mathrm{LNCaP}$ and PC-3 cells for which $\mathrm{IC}_{50}$ values were extrapolated to be $2.20 \pm 0.32$ and $3.10 \pm 0.16 \mu \mathrm{mol} / \mathrm{L}$, respectively $(P<0.05$, ANOVA $)$. 


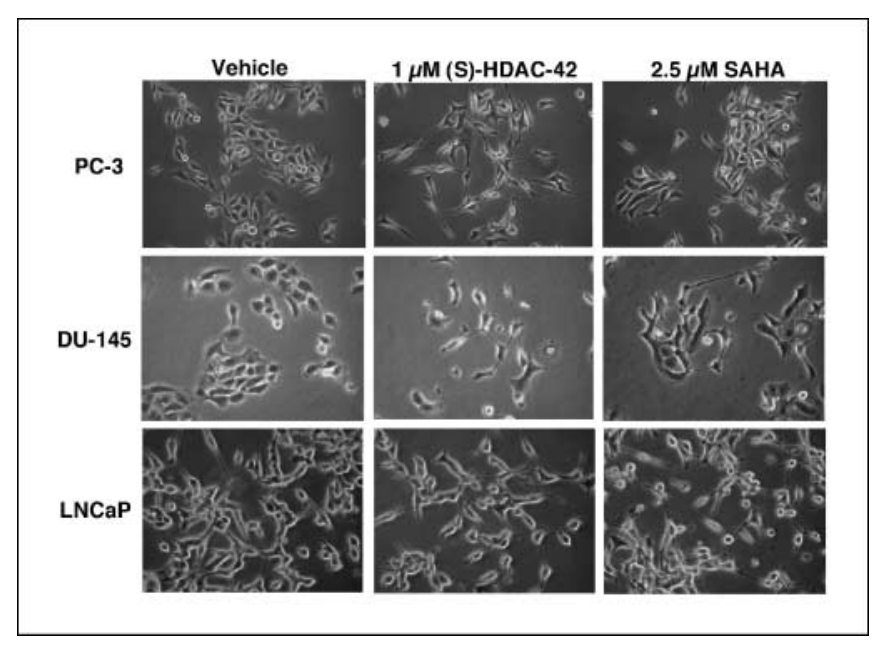

Fig. 2. Morphologic changes in PC-3, DU-145 and LNCaP cells treated with vehicle control (left), $1 \mu \mathrm{mol} / \mathrm{L}(S)$-HDAC-42 (middle), or $2.5 \mu \mathrm{mol} / \mathrm{L} \mathrm{SAHA} \mathrm{(right)}$ for 24 hours. Original magnification, $\times 100$.

This discrepancy in cellular sensitivity might reflect the differences in mechanisms underlying the antiproliferative effects of these two agents as described below. Assessment of effects on nonmalignant cells revealed that PrECs exhibited a 7.5- to 9.5fold lower sensitivity to the antiproliferative effects of $(S)$ HDAC-42 than the prostate cancer cells $\left(\mathrm{IC}_{50}, 3.69 \pm 0.96\right.$ $\mu \mathrm{mol} / \mathrm{L} ; P<0.001$, ANOVA; Fig. 1C).

It is noteworthy that although neither agent showed a sizeable inhibition of cell proliferation at 24 hours of treatment, particularly in PC-3 cells, morphologic changes were evident. As shown in Fig. 2, 24 hours' exposure of PC-3 cells to $1 \mu \mathrm{mol} / \mathrm{L}(S)$-HDAC-42 transformed the typical epithelial morphology to a more spindle-shaped morphology (top left and middle). These drug-treated cells seemed to be severely contracted with diminished cell-to-cell contacts and long spine-like processes. Similar morphologic changes were also observed in PC-3 cells treated with $2.5 \mu \mathrm{mol} / \mathrm{L}$ of SAHA, although to a lesser extent (top right). These changes were also apparent in DU-145 (middle) and LNCaP cells (bottom), although the typical spindle-like morphology of untreated LNCaP cells made such changes in them more difficult to discern. Although the driving force underlying these morphologic changes remain unclear, these findings indicate a clear effect of drug treatment on prostate cancer cells, which preceded the observable effects on cell viability and/or apoptosis.

Western blot analysis of the status of p21 expression and histone H3 acetylation in drug-treated cells revealed dosedependent up-regulation of these HDAC-associated biomarkers (Fig. 3A), which paralleled the drugs' activities in inhibiting cell proliferation. Of these two HDAC inhibitors, ( $S$ )-HDAC-42 exhibited higher potency in stimulating p21 up-regulation and histone H3 hyperacetylation (right). In addition, as evidenced by mitochondrial cytochrome $c$ release, activation of caspase 9, cleavage of PARP (Fig. 3A, left), and DNA fragmentation (Fig. 3B), the antiproliferative activity of (S)-HDAC-42 was, at least in part, attributable to apoptosis. Moreover, these changes in apoptotic markers were markedly higher than those observed in the SAHA-treated PC-3 cells, consistent with the greater potency of $(S)$-HDAC-42.
(S)-HDAC-42 dephosphorylates Akt and alters the dynamics of Bcl-xL expression. Previously, we reported that (S)-HDAC-42 and, to a lesser extent, SAHA caused Akt dephosphorylation in PC-3 cells through the disruption of HDAC-protein phosphatase (PP1) complexes, leading to increased PP1-Akt association (18). This finding indicates the complexity in the mechanism of $(S)$-HDAC-42's antitumor effects and suggests a basis for the difference in the apoptogenic potencies of these two compounds. Moreover, the modulation of the expression of Bcl-2 family members by several HDAC inhibitors has been reported in a variety of cancer cell lines including mesothelioma, prostate cancer, melanoma, leukemia, and lung cancer $(32-36)$. To shed light into the mechanism underlying the differential antiproliferative activities of $(S)$-HDAC-42 and SAHA, the effects of these agents on the status of Akt signaling and expression levels of Bcl-2 family members in PC-3 cells were compared.

Western blot analysis revealed that $(S)$-HDAC-42, even at $1 \mu \mathrm{mol} / \mathrm{L}$, caused substantial reductions in the levels of p- ${ }^{473}$ Ser- and p- ${ }^{308}$ Thr-Akt $(64.4 \pm 11.9 \%$ and $61.4 \pm 14.8 \%$ reductions, respectively, compared with DMSO-treated controls) in PC-3 cells after 72 hours of exposure. In contrast, SAHA required at least $5 \mu \mathrm{mol} / \mathrm{L}$ to attain similar levels of Akt dephosphorylation $(48.9 \pm 12.7 \%$ and $58.0 \pm 15.6 \%$, respectively; Fig. 4B). To validate the functional consequence of diminished phospho-Akt, we examined the phosphorylation status of Bad, an apoptosis-relevant substrate of Akt. In parallel with phospho-Akt levels, the amount of phospho-Bad was significantly reduced by $(S)$-HDAC-42 without changes in the

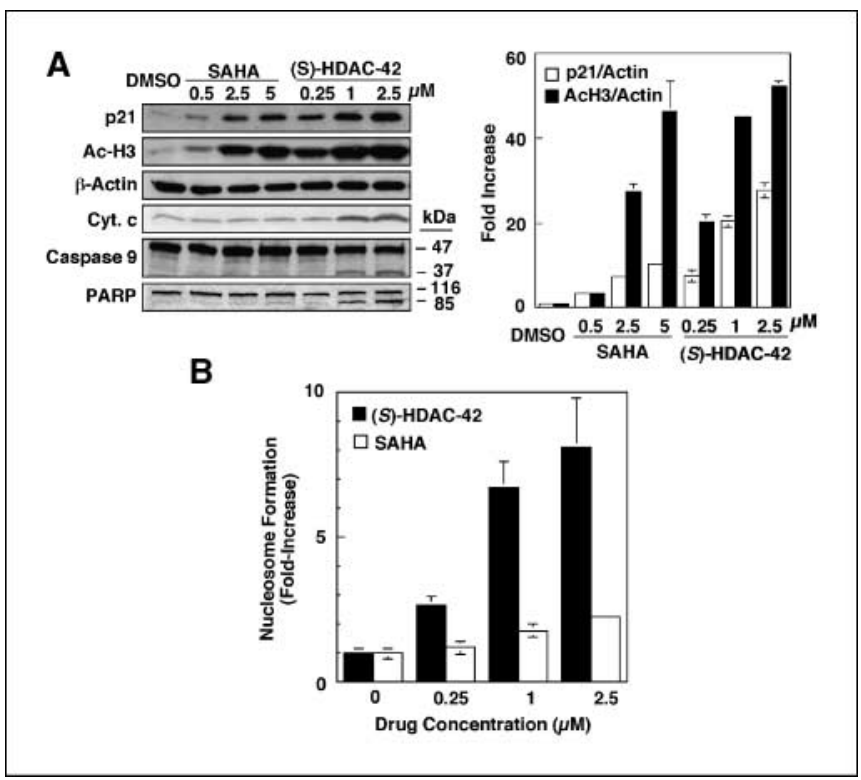

Fig. 3. Effects of $(S)$-HDAC-42 versus SAHA on various biomarkers associated with HDAC inhibition or apoptosis in PC-3 cells. $A$, Western blot analysis of the dose-dependent effects of $(S)$-HDAC-42 and SAHA on p21 expression, histone H3 acetylation, cytochrome $c$ release, caspase 9 activation, and PARP cleavage in PC-3 cells after 72 hours' exposure in 10\% FBS-supplemented RPMI 1640. A representative immunoblot (left). Signals for $\mathrm{p} 21$ and acetylated histone $\mathrm{H} 3$ were quantitated by densitometry and normalized against that of $\beta$-actin (right).

Columns, mean; bars, $\pm \mathrm{SD}(n=3)$. AcH3, acetylated histone H3. $B$, formation of cytoplasmic nucleosomal DNA in PC-3 cells treated with $(S)$-HDAC-42 or SAHA at the indicated concentrations for 72 hours in 10\% FBS-supplemented RPMI 1640 DNA fragmentation was quantitatively measured by a cell death detection ELISA kit as described in Materials and Methods. Columns, mean; bars, $\pm \operatorname{SD}(n=3)$. 


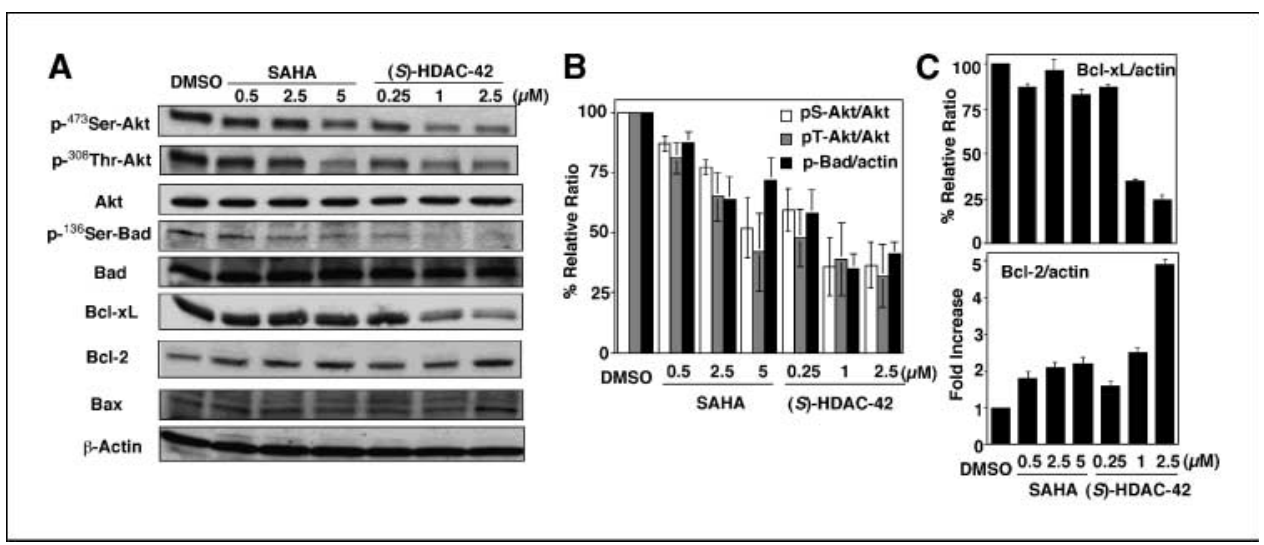

Fig. 4. Dose-dependent effects of $(S)$-HDAC-42 versus SAHA on the phosphorylation state of ${ }^{473}$ Ser- and ${ }^{308}$ Thr-Akt and ${ }^{136}$ Ser-Bad, and the expression levels of Bad, $\mathrm{Bcl}-\mathrm{xL}, \mathrm{Bcl}-2$, and Bax in PC-3 cells. The cells were treated with the indicated concentrations of SAHA or (S)-HDAC-42 in $10 \%$ FBS-containing RPMI 1640 for 72 hours, and cell lysates were immunoblotted as described in Materials and Methods. $A$, representative immunoblot. $B$, relative expression levels of $\mathrm{p}-{ }^{473}$ Ser-Akt, $\mathrm{p}-{ }^{308}$ Thr-Akt, and $\mathrm{p}-{ }^{136}$ Ser-Bad. Amounts of immunoblotted proteins were quantitated by densitometry and normalized to those of total Akt or $\beta$-actin. Columns, mean; bars, $\pm \mathrm{SD}(n=3)$. pS-Akt, p- ${ }^{473}$ Ser-Akt; pT-Akt, p- ${ }^{308}$ Thr-Akt; p-Bad, $\mathrm{p}^{-136}$ Ser-Bad. C, relative expression levels of Bcl-xL (top) and Bcl-2 (bottom). Amounts of immunoblotted proteins were quantitated by densitometry and normalized to that of $\beta$-actin. Columns, mean; bars, $\pm \operatorname{SD}(n=3)$.

expression of total Bad protein (Fig. 4A and B). Furthermore, $(S)$-HDAC-42 caused a pronounced, dose-dependent attenuation in Bcl-xL expression, achieving a $75.4 \pm 2.5 \%$ reduction at $2.5 \mu \mathrm{mol} / \mathrm{L}$. In contrast, SAHA failed to reduce Bcl-xL protein levels below $82.8 \pm 2.8 \%$ of the control level at the highest concentration tested (Fig. 4C, top). This clear difference in the abilities of SAHA and (S)-HDAC-42 to suppress Bcl-xL protein expression was mirrored by their respective effects on steady state levels of $\mathrm{Bcl}-\mathrm{xL}$ mRNA. Semiquantitative reverse transcription-PCR revealed that SAHA reduced Bcl-xL mRNA levels by $9.5 \pm 1.7 \%, 9.3 \pm 1.3 \%$, and $10.2 \pm 6.0 \%$ at 0.5 , 2.5 , and $5.0 \mu \mathrm{mol} / \mathrm{L}$, respectively, compared with vehicletreated controls, whereas $(S)$-HDAC-42 more potently suppressed mRNA levels by $16.7 \pm 9.0 \%, 38.9 \pm 3.3 \%$, and $46.4 \pm$ $4.4 \%$ at $0.25,1.0$, and $2.5 \mu \mathrm{mol} / \mathrm{L}$, respectively. ( $S$ )-HDAC-42 also caused a nearly 2-fold increase in Bax expression level, whereas no detectable increase occurred in SAHA-treated PC-3 cells (Fig. 4A). These proapoptotic changes in Bcl-2 family members were accompanied by increases in the expression levels of antiapoptotic Bcl-2 protein in cells treated with either agent. (S)-HDAC-42 treatment induced a 1.6- to 4.9-fold increase in the amount of Bcl-2 protein over the concentration range tested, whereas SAHA caused a 2.2-fold increase at $5 \mu \mathrm{mol} / \mathrm{L}$ (Fig. 4C, bottom).

(S)-HDAC-42 attenuates protein levels of IAP family members. Members of the IAP family of proteins have been implicated in oncogenesis, cancer progression, and therapeutic resistance, at least in part, through their well-known antiapoptotic function of inhibiting caspase activity $(37,38)$. Several reports have described the suppression of IAP family members by treatment with HDAC inhibitors in cancer cells $(35,36,39-43)$, as well as tumor endothelium (41). Accordingly, we sought to determine the effects of $(S)$-HDAC42 and SAHA on the suppression of IAP members, including survivin, CIAP-1, cIAP-2, and ILP, in PC-3 cells. As shown in Fig. 5A and $\mathrm{B},(S)$-HDAC-42 induced a profound, dosedependent loss of survivin that ranged from a $64.6 \pm 6.2 \%$ reduction at $0.25 \mu \mathrm{mol} / \mathrm{L}$ up to $93.6 \pm 2.5 \%$ at $2.5 \mu \mathrm{mol} / \mathrm{L}$. SAHA caused a similar, although less potent, effect on survivin expression, diminishing it at all concentrations tested up to a $64.1 \pm 7.6 \%$ reduction after treatment with $5.0 \mu \mathrm{mol} / \mathrm{L}$. Although less robust, decrements in survivin were also observed at the level of mRNA. The highest concentrations of ( $S$ )-HDAC42 and SAHA tested ( 2.5 and $5.0 \mu \mathrm{mol} / \mathrm{L}$, respectively) reduced steady-state levels of survivin mRNA to $67.1 \pm 1.7 \%$ and $81.9 \pm 1.1 \%$ of that of the vehicle-treated controls. Of the other IAPs evaluated (Fig. 5A), cIAP-2 was the most responsive to either drug exhibiting 1.7 - and 2.5-fold reductions after treatment with 5.0 and $2.5 \mu \mathrm{mol} / \mathrm{L}$ of SAHA and $(S)$-HDAC42 , respectively. $(S)$-HDAC-42 also induced a 2 -fold inhibition of cIAP-1 at $2.5 \mu \mathrm{mol} / \mathrm{L}$; however, neither agent altered the expression of ILP.
Fig. 5. Dose-dependent effects of $(S)$-HDAC-42 versus SAHA on the expression levels of members of the IAP family of proteins including survivin, cIAP-1, cIAP-2, and ILP, in PC- 3 cells. The cells were treated with the indicated concentrations of SAHA or $(S)$-HDAC-42 in $10 \%$ FBS-containing RPMI 1640 for 72 hours, and cell lysates were immunoblotted as described in Materials and Methods. $A$, representative immunoblot. $B$, relative expression levels of survivin. Amounts of immunoblotted proteins were quantitated by densitometry and normalized to that of $\beta$-actin. Columns, mean; bars, $\pm \mathrm{SD}(n=3)$.

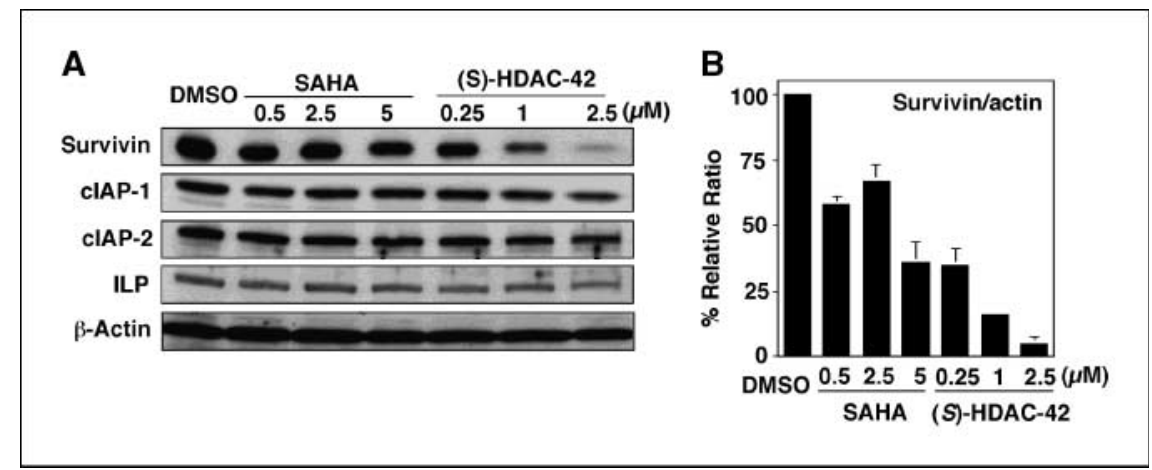




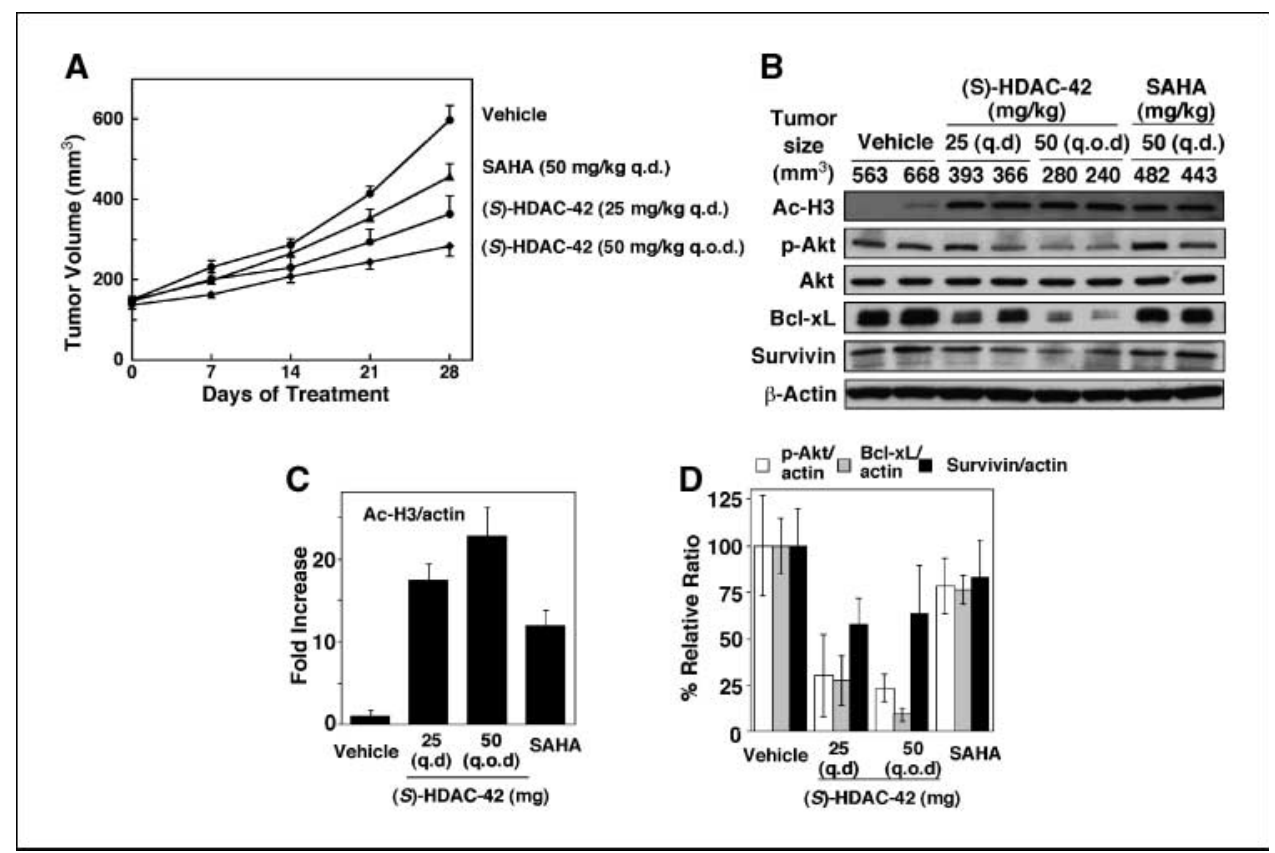

Fig. 6. Effects of oral (S)-HDAC-42 at $25 \mathrm{mg} / \mathrm{kg}$ per day (q.d.) or $50 \mathrm{mg} / \mathrm{kg}$ every other day (q.o.d.) versus oral SAHA at $50 \mathrm{mg} / \mathrm{kg}$ per day on the growth of established PC-3 tumors in nude mice and the expression of intratumoral biomarkers of drug activity. Each mouse was inoculated s.c. in the right flank with $5 \times 10^{5}$ PC- 3 cells in a total volume of $0.1 \mathrm{~mL}$ serum-free medium containing $50 \%$ Matrigel. Mice with established tumors (mean tumor volume, $145.1 \pm 22.1 \mathrm{~mm}^{3}$ ) were randomized to four groups $(n=7)$ that received individual treatments by gavage for the duration of the study as described under Materials and Methods. $A$, mean tumor volumes for each treatment group as a function of day of treatment. Points, mean tumor volume of seven animals; bars, $\pm \mathrm{SE}$. $B$, Western blot analysis of intratumoral biomarkers of drug activity in the homogenates of two representative PC-3 tumors from each treatment group and their respective final volumes. $C$, relative expression levels of acetylated histone $\mathrm{H} 3$.

Amounts of immunoblotted proteins from all available tumor lysates were quantitated by densitometry and normalized to that of $\beta$-actin. Columns, mean; bars, $\pm \mathrm{SD}(n=6)$. $D$, relative expression levels of phospho-Akt, $\mathrm{Bcl}-\mathrm{xL}$, and survivin. Amounts of immunoblotted proteins from all available tumor lysates were quantitated by densitometry and normalized to that of total Akt or $\beta$-actin. Columns, mean; bars, $\pm \operatorname{SD}(n=6)$.

(S)-HDAC-42 suppresses prostate tumor xenograft growth in vivo. Collectively, the in vitro data described above indicate that $(S)$-HDAC-42 is a potent inhibitor of prostate cancer cell proliferation and survival, and owes this antitumor efficacy to the modulation of apoptotic machinery at multiple levels, including Akt signaling, mitochondrial integrity, and caspase activation. To further evaluate the antitumor potential of $(S)$-HDAC-42, athymic nude mice bearing established s.c. PC3 tumor xenografts (mean tumor volume, $145.1 \pm 22.1 \mathrm{~mm}^{3}$ ) were treated orally for 28 days with $(S)$-HDAC-42 at $25 \mathrm{mg} / \mathrm{kg}$ daily or $50 \mathrm{mg} / \mathrm{kg}$ every other day, with SAHA at $50 \mathrm{mg} / \mathrm{kg}$ daily, or with vehicle. As shown in Fig. 6A, treatment of mice with SAHA, $25 \mathrm{mg} / \mathrm{kg}$ of (S)-HDAC-42 daily and $50 \mathrm{mg} / \mathrm{kg}$ of $(S)$-HDAC-42 every other day significantly inhibited PC-3 tumor growth by $31 \%, 52 \%$, and $67 \%$, respectively, relative to vehicle-treated controls $(P<0.01)$. Moreover, at $50 \mathrm{mg} / \mathrm{kg}$ every other day, the antitumor effect of $(S)$-HDAC-42 was significantly greater than that of SAHA at $50 \mathrm{mg} / \mathrm{kg}$ daily $(P<0.001)$. This in vivo efficacy after oral administration confirmed $(S)$ HDAC-42's oral bioavailability, which was previously determined to be $26 \%{ }^{3}$ Importantly, mice seemed to tolerate all of the agents without overt signs of toxicity, without significant changes in body weight compared with the vehicle-treated group, and without abnormalities in serum chemistry variables. The sole gross and histopathologic findings associated with $(S)$ -

\footnotetext{
${ }^{3}$ Compound summary, January 4, 2006, NCI RAID Initiative for NSC D735012, contract no. N01-CM-52205
}

HDAC-42 treatment were testicular atrophy and a marked diffuse testicular degeneration with associated epididymal hypospermia or aspermia. These testicular changes were not observed in the SAHA-treated mice.

To correlate this in vivo tumor-suppressive response to mechanisms identified in vitro, the effects of treatment with HDAC inhibitors on intratumoral biomarkers of drug activity were evaluated by immunoblotting of PC-3 tumor homogenates collected after 28 days of treatment. As shown in Fig. 6B and C, treatment of mice with $(S)$-HDAC-42 or SAHA increased levels of acetylated histone $\mathrm{H} 3$ in $\mathrm{PC}-3$ tumor xenografts relative to the vehicle-treated controls, thus confirming HDAC inhibition in vivo. The effects of these agents on Akt dephosphorylation and Bcl-xL down-regulation approximated those observed in vitro, which reflected their differential tumor suppressive activities in vivo. Treatment with (S)-HDAC-42 at $25 \mathrm{mg} / \mathrm{kg}$ per day and $50 \mathrm{mg} / \mathrm{kg}$ every other day induced marked reductions in intratumoral levels of both phospho-Akt $(69.8 \pm 22.1 \%$ and $76.8 \pm 7.4 \%$ reductions, respectively, compared with vehicle-treated controls) and Bcl-xL (72.6 $\pm 13.2 \%$ and $90.9 \pm 3.4 \%$ reductions, respectively; Fig. 6 D). SAHA treatment $(50 \mathrm{mg} / \mathrm{kg} / \mathrm{d})$, however, caused much smaller changes in these biomarkers, reducing phospho-Akt by $21.6 \pm 15 \%$ and Bcl-xL by $23.6 \pm 7.8 \%$ (Fig. 6D). In contrast to the marked suppression of survivin by $(S)$-HDAC-42 and SAHA in vitro (Fig. 5B), its in vivo suppression by these agents was less dramatic. ( $S$ )-HDAC-42 at $25 \mathrm{mg} / \mathrm{kg}$ per day and $50 \mathrm{mg} / \mathrm{kg}$ every other day reduced survivin expression by $42.8 \pm 14.5 \%$ and $56.5 \pm 25.6 \%$, respectively, whereas SAHA diminished it by only $17.3 \pm 20.3 \%$ (Fig. 6D). 


\section{Discussion}

HDAC is recognized as one of the promising targets for cancer treatment because many HDAC inhibitors have entered clinical trials for both solid and liquid tumors. In this report, we describe the in vitro and in vivo efficacy of a novel phenylbutyrate-derived HDAC inhibitor, $(S)$-HDAC-42, in prostate cancer. Our findings show that $(S)$-HDAC-42 exhibits a broad spectrum of antitumor activities at low micromolar concentrations that involve not only histone acetylation, but also the modulation of apoptotic regulators at multiple levels, including Akt signaling, mitochondrial integrity, and caspase activation.

In comparison with SAHA, a HDAC inhibitor currently in clinical trials, $(S)$-HDAC-42 exhibited greater antiproliferative activity in multiple prostate cancer cell lines in vitro. The greater potency of $(S)$-HDAC-42 to induce apoptosis, as evidenced by cytochrome $c$ release, activation of caspase 9, PARP cleavage, and DNA fragmentation seemed to underlie this higher antitumor efficacy. Moreover, the marked differences in apoptosis induction between these two agents were paralleled by similar differences in their relative abilities to decrease Akt phosphorylation, to stimulate Bax expression, and to suppress expression levels of survivin and particularly Bcl-xL in vitro. This ability of (S)-HDAC-42 to affect multiple regulators of cancer cell survival is reflected in its equipotent antiproliferative effects against three prostate cancer cells lines that differ with respect to the functional status of p53, PTEN, and Bcl-xL expression levels, and suggests its potential clinical efficacy against molecularly heterogeneous tumors.

Evidence indicates that whereas histones still represent a primary target for the physiologic function of HDACs, the antitumor effects of some HDAC inhibitors, including $(S)$ HDAC-42, might also be attributed to histone acetylationindependent mechanisms by interfering with the activation or expression status of a number of signaling targets (19). Previously, we have shown that $(S)$-HDAC-42 mediates Akt dephosphorylation through the disruption of PP1-HDAC complexes, resulting in increased PP1-Akt association (18). Whether the observed (S)-HDAC-42-induced repression of $\mathrm{Bcl}-\mathrm{xL}$ and survivin also occurred independently of histone acetylation cannot be concluded definitively from our study. Moreover, because levels of phospho-Akt and survivin were also reduced, although to a lesser degree, by SAHA treatment, it is likewise unclear whether the differential effects of these agents on apoptotic regulators are merely a function of their different potencies and/or result from true differences in the spectrum of their respective pharmacologic targets.

The correlation between the differential modulation of apoptotic signaling targets and in vitro antitumor activities of $(S)$-HDAC-42 and SAHA was mirrored in our in vivo study, in which $(S)$-HDAC-42 exhibited higher potency than SAHA in suppressing established PC-3 xenograft tumor growth. Western blot analysis of the tumor lysates revealed that the greater tumor growth inhibition of $(S)$-HDAC-42 paralleled its greater ability to inhibit putative histone acetylation-independent biomarkers, particularly Akt phosphorylation and $\mathrm{Bcl}-\mathrm{xL}$ expression. With the exception of the observed testicular pathology, tumor growth suppression by (S)-HDAC-42 occurred in the absence of limiting toxicity. Although testicular toxicities are common adverse side effects of cancer therapy (44), to our knowledge, investigators have not previously reported testicular pathology as a consequence of HDAC inhibitor treatment in preclinical models of human cancer. A single study examining the antifertility effect of trichostatin A in mice reported a completely reversible impairment of spermatogenesis resulting from increased apoptosis of spermatocytes (45). Although we do not know the cellular target mediating this toxicity of $(S)$-HDAC-42, the lack of pathologic changes in the pituitary and accessory sex organs suggests a primary testicular toxicity. Supporting this supposition is the absence of histopathologic changes in the gut and bone marrow, which are also common sites of adverse chemotherapeutic consequences.

In conclusion, our results show that the novel orally bioavailable, phenylbutyrate-derived HDAC inhibitor, $(S)$ HDAC-42, is a potent inhibitor of HDAC, as well as targets regulating multiple aspects of cancer cell survival including Akt signaling, mitochondrial integrity, and caspase activity. This broad spectrum of activity underlies the more potent apoptogenic and antitumor activities of $(S)$-HDAC-42 in vitro and in vivo relative to SAHA, and suggests its viability as part of a therapeutic strategy for prostate cancer.

\section{References}

1. Cress WD, Seto E. Histone deacetylases, transcriptional control, and cancer. J Cell Physiol 2000;184:1 -16.

2. Marks P, Rifkind RA, Richon VM, Breslow R, Miller T, Kelly WK. Histone deacetylases and cancer: causes and therapies. Nat Rev Cancer 2001;1:194-202.

3. Johnstone RW. Histone-deacetylase inhibitors: novel drugs for the treatment of cancer. Nat Rev Drug Discov 2002;1:287-99.

4. McLaughlin F, LaThangue NB. Histone deacetylase inhibitors open new doors in cancer therapy. Biochem Pharmacol 2004:68:1139-44.

5. Minucci S, Pelicci PG. Histone deacetylase inhibitors and the promise of epigenetic (and more) treatments for cancer. Nat Rev Cancer 2006:6:38-51.

6. Jung M. Inhibitors of histone deacetylase as new anticancer agents. Curr Med Chem 2001;8:1505-11.

7. Wade PA. Transcriptional control at regulatory checkpoints by histone deacetylases: molecular connections between cancer and chromatin. Hum Mol Genet 2001;10:693-8.

8. Glaser KB, Staver MJ, Waring JF, Stender J, Ulrich RG, Davidsen SK. Gene expression profiling of multi- ple histone deacetylase (HDAC) inhibitors: defining a common gene set produced by HDAC inhibition in T24 and MDA carcinoma cell lines. Mol CancerTher 2003; 2:151-63.

9. Villar-Garea A, Esteller M. Histone deacetylase inhibitors: understanding a new wave of anticancer agents. Int J Cancer 2004;112:171 -8.

10. Johnstone RW, Licht JD. Histone deacetylase inhibitors in cancer therapy: is transcription the primary target? Cancer Cell 2003;4:13-8.

11. Chen L, Fischle W, Verdin E, Greene WC. Duration of nuclear NF- $\kappa \mathrm{B}$ action regulated by reversible acetylation. Science 2001;293:1653-7.

12. Chen LF, Greene WC. Regulation of distinct biological activities of the NF- $\kappa$ B transcription factor complex by acetylation. J Mol Med 2003;81:549-57.

13. Yuan ZL, Guan YJ, Chatterjee D, Chin YE. Stat3 dimerization regulated by reversible acetylation of a single lysine residue. Science 2005;307:269-73.

14. Bode AM, Dong Z. Post-translational modification of p53 in tumorigenesis. Nat Rev Cancer 2004;4: $793-805$.
15. Roy S, Packman K, Jeffrey R, Tenniswood M Histone deacetylase inhibitors differentially stabilize acetylated p53 and induce cell cycle arrest or apoptosis in prostate cancer cells. Cell Death Differ 2005;12: 482-91.

16. Fuino $L$, Bali $P$, Wittmann $S$, et al. Histone deacetylase inhibitor LAQ824 down-regulates Her-2 and sensitizes human breast cancer cells to trastuzumab, taxotere, gemcitabine, and epothilone B. Mol Cancer Ther 2003;2:971-84.

17. Chen $\mathrm{L}$, Meng $\mathrm{S}$, Wang $\mathrm{H}$, et al. Chemical ablation of androgen receptor in prostate cancer cells by the histone deacetylase inhibitor LAQ824. Mol Cancer Ther 2005;4:1311 -9.

18. Chen CS, Weng SC, Tseng PH, Lin HP. Histone acetylation-independent effect of histone deacetylase inhibitors on Akt through the reshuffling of protein phosphatase 1 complexes. J Biol Chem 2005;280: 38879-87.

19. Lin HY, Chen CS, Lin SP, Weng JR, Chen CS. Targeting histone deacetylase in cancer therapy. Med Res Rev 2006:26:397-413. 
20. Lu Q, Yang YT, Chen CS, et al. Zn2+-chelating motif-tethered short-chain fatty acids as a novel class of histone deacetylase inhibitors. J Med Chem 2004; 47:467-74

21. Lu Q, Wang DS, Chen CS, HuYD. Structure-based optimization of phenylbutyrate-derived histone deacetylase inhibitors. J Med Chem 2005;48:5530-5.

22. Patra SK, Patra A, Dahiya R. Histone deacetylase and DNA methyltransferase in human prostate cancer. Biochem Biophys Res Commun 2001;287:705-13.

23. Halkidou $K$, Cook S, Leung HY, Neal DE, Robson CN. Nuclear accumulation of histone deacetylase 4 (HDAC4) coincides with the loss of androgen sensitivity in hormone refractory cancer of the prostate [author reply 9]. Eur Urol 2004;45:382-9.

24. Halkidou K, Gaughan L, Cook S, Leung HY, Nea $\mathrm{DE}$, Robson CN. Upregulation and nuclear recruitmen of HDAC1 in hormone refractory prostate cancer. Prostate 2004;59:177-89.

25. Li LC, Carroll PR, Dahiya R. Epigenetic changes in prostate cancer: implication for diagnosis and treatment. J Natl Cancer Inst 2005:97:103-15.

26. Butler LM, Agus DB, Scher HI, et al. Suberoylanilide hydroxamic acid, an inhibitor of histone deacetylase, suppresses the growth of prostate cancer cells in vitro and in vivo. Cancer Res 2000;60:5165-70.

27. Rashid SF, Moore JS, Walker E, et al. Synergistic growth inhibition of prostate cancer cells by $1 \alpha 25$ dihydroxyvitamin $\mathrm{D}(3)$ and its 19-nor-hexafluorid analogs in combination with either sodium butyrate or trichostatin A. Oncogene 2001:20:1860-72.

28. Kuefer R, Hofer MD, Altug V, et al. Sodium butyrate and tributyrin induce in vivo growth inhibition and apoptosis in human prostate cancer. $\mathrm{Br} \mathrm{J}$ Cance 2004:90:535-41.

29. Fronsdal K, Saatcioglu F. Histone deacetylase inhibitors differentially mediate apoptosis in prostate cancer cells. Prostate 2005:62:299-306.

30. Chou TC, Talalay P. Quantitative analysis of doseeffect relationships: the combined effects of multiple drugs or enzyme inhibitors. Adv Enzyme Regul 1984; 22:27-55

31. Kulp SK, Yang YT, Hung CC, et al. 3-Phosphoinositide-dependent protein kinase-1/Akt signaling represents a major cyclooxygenase-2-independent target for celecoxib in prostate cancer cells. Cancer Res 2004:64:1444-51.

32. Cao XX, Mohuiddin I, Ece F, McConkey DJ, SmytheWR. Histone deacetylase inhibitor downreguation of bcl-xl gene expression leads to apoptotic cell death in mesothelioma. Am J Respir Cell Mol Biol 2001;25:562-8.

33. Goh M, Chen F, Paulsen MT, Yeager AM, Dyer ES, Ljungman $M$. Phenylbutyrate attenuates the expression of $\mathrm{Bcl}-\mathrm{X}(\mathrm{L})$, DNA-PK, caveolin-1, and VEGF in prostate cancer cells. Neoplasia 2001;3:331 -8.

34. Zhang XD, Gillespie SK, Borrow JM, Hersey P. The histone deacetylase inhibitor suberic bishydroxamate regulates the expression of multiple apoptotic mediators and induces mitochondria-dependent apoptosis of melanoma cells. Mol CancerTher 2004;3:425-35.

35. Guo F, Sigua $C$, Tao J, et al. Cotreatment with histone deacetylase inhibitor LAQ824 enhances Apo$2 \mathrm{~L} /$ tumor necrosis factor-related apoptosis inducing igand-induced death inducing signaling complex activity and apoptosis of human acute leukemia cells. Cancer Res 2004;64:2580-9.

36. Doi S, Soda H, Oka M, et al. The histone deacetylase inhibitor FR901228 induces caspase-dependent apoptosis via the mitochondrial pathway in small cell lung cancer cells. Mol Cancer Ther 2004. 3:1397-402
37. Zaffaroni N, Pennati M, Daidone MG. Survivin as a target for new anticancer interventions. J Cell Mol Med 2005:9:360-72

38. Wright CW, Duckett CS. Reawakening the cellular death program in neoplasia through the therapeutic blockade of IAP function. J Clin Invest 2005;115: 2673-8.

39. Mitsiades N, Mitsiades CS, Richardson PG, et al. Molecular sequelae of histone deacetylase inhibition in human malignant B cells. Blood 2003;101: 4055-62.

40. De Schepper S, Bruwiere H, Verhulst T, et al. Inhibition of histone deacetylases by chlamydocin induces apoptosis and proteasome-mediated degradation of survivin. J Pharmacol ExpTher 2003;304:881 -8.

41. Qian DZ, Wang $X$, Kachhap $S K$, et al. The histone deacetylase inhibitor NVP-LAQ824 inhibits angiogenesis and has a greater antitumor effect in combination with the vascular endothelial growth factor receptor tyrosine kinase inhibitor PTK787/ZK222584. Cancer Res 2004:64:6626-34.

42. Maggio SC, Rosato RR, Kramer LB, et al. The histone deacetylase inhibitor MS-275 interacts synergistically with fludarabine to induce apoptosis in human leukemia cells. Cancer Res 2004;64:2590-600.

43. Sato N, Ohta T, Kitagawa H, et al. FR901228, a novel histone deacetylase inhibitor, induces cell cycle arrest and subsequent apoptosis in refractory human pancreatic cancer cells. Int J Oncol 2004:24:679-85. 44. Boekelheide K. Mechanisms of toxic damage to spermatogenesis. J Natl Cancer Inst Monogr 2005; 34:6-8.

45. Fenic I, Sonnack V, Failing K, Bergmann M, Steger $\mathrm{K}$. In vivo effects of histone-deacetylase inhibitor trichostatin-A on murine spermatogenesis. J Androl 2004;25:811-8. 